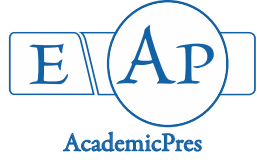

\title{
Morphological, Anatomical and Cytological Studies of some Moss Species from Nigeria
}

\author{
Abolade Oluremi BOLAJI*, Julius Olaoye FALUYI \\ Obafemi Awolowo University, Faculty of Science, Department of Botany, Ile-Ife, \\ Nigeria; aboladebolaji2017@gmail.com (*corresponding author);jfaluyi@gmail.com
}

\begin{abstract}
Morphological, anatomical and chromosome studies of Hyophila crenulata C, Mull. Ex Dus, Thuidium gratum (P. Beauv) Jaeg., Barbula lambarenensis P. Vard., Stereophyllum nitense Mitt. and Bryum coronatum Schwaegr from Nigeria, were carried out with a view to bridging some knowledge gaps that exist in their characterization and providing insightful information that could be useful in elucidating their taxonomic status. The morphological and anatomical studies revealed several gametophytic and sporophytic attributes which have not been previously reported and which were diagnostic for the moss species studied. The chromosome studies revealed the chromosome numbers to be Hyophila crenulata $n=4$; Thuidium gratum $n=12(10+2$ $\mathrm{m})$; Barbula lambarenensis $n=3$; Stereophyllum nitense $n=9$; and Bryum coronatum $n=10$. From the results of the study, it could be concluded that the details of the morphological and anatomical descriptions as well as the chromosome numbers being reported for the first time in this study for the moss species studied could be very useful in their identification and taxonomic delimitation.
\end{abstract}

Keywords: anatomical features, bryophytes, chromosomal complement, gametophyte, sporophyte

\section{Introduction}

Mosses are non-vascular plants that occur over a wide range of habitats such as rocks, soil, logs, tree trunks and concrete walls. They constitute a group in the division bryophyta, the other two groups in the division being Marchantiophyta (liverworts) and Anthocerotophyta also known as hornworts (Buck and Goffinet, 2000; Adebiyi $e t$ al., 2012; Oyesiku, 2012).

Mosses play significant ecological role in the ecosystem (Saxena and Harrinder, 2004). They play important role in soil formation and plant succession (Aline et al., 2012). They are widely used as medicinal plants and their antimicrobial properties are very useful in herbal medicines (Dulger et al., 2005; Iihan et al., 2006; Ojo et al., 2007; Bobade et al., 2008; Russell, 2010; Elibol et al., 2011; Savoroglu et al., 2011). They also serve as food for a wide range of animals (Davidson et al., 1990; Dickson, 1997).

Apart from Bryum coronatum which is geographically wide spread, the other four species selected for this study namely Hyophila crenulata, Thuidium gratum, Barbula lambarenensis and Stereophyllum nitense are endermic to Nigeria and are particularly of interest to many researchers because of their phytochemical, antimicrobial and insecticidal activities (Ande et al., 2010; Adebiyi et al., 2012; Adebiyi et al., 2013; Femi-Adepoju et al., 2014; Tedela et al., 2014), as well as their effectiveness as bio-indicators in the monitoring of environmental pollution (Batagarawa and Lawal, 2010; Ekpo et al., 2012; Ite et al., 2014).

Much is still left to be done in the characterization of these speceies as information about their morphological and anatomical attributes is quite sparse and limited to a few information that emanated from the works of Egunyomi (1979, 1980, 1981, 1984), Egunyomi and Vital (1984), Makinde and Odu (1993) and Fatoba and Odu, (1999) leaving knowledge gaps that has sometimes led to misidentification of some of these species by researchers. Many aspects of the morphological and anatomical attributes of species selected for this study are yet to be investigated and reported. These include aspects such as the morphological and anatomical characterization of the leaf cells, capsules, peristomes, operculum and spores. Also, there is no known record of the chromosome number of any of these moss species from Nigeria.

The objectives of this study are to characterize samples of some Nigerian moss species using morphological and anatomical parameters many of which have not been documented; and also established the chromosome numbers of these moss species for the first time. 


\section{Materials and Methods}

\section{Plant source and identification}

The moss species investigated were Hyophila crenulata C, Mull. Ex Dus, Thuidium gratum (P. Beauv) Jaeg., Barbula lambarenensis P. Vard. and Stereophyllum nitense Mitt. Samples were collected from various locations in IleIfe, Nigeria (Table 1), during the raining seasons between April 2014 and November 2015) while the plants were in full bloom and in their optimal conditions. Samples collected were identified at the herbarium of the University of Ibadan, Nigeria.

\section{Morphological studies}

The gametophytic as well as sporophytic attributes of the different species of mosses collected were characterized by morphological description according to Smith (1978) and measurements were made with the aid of the light microscope, dissecting microscope and ocular micrometer.

Characters investigated include: plant habitat, plant colour when moist and when dry, perichaetial leaf shape, perichaetial leaf apex shape, perichaetial leaf margin shape, shape of lamina cells at the apex, middle and basal portion of the perichaetial leaves, shape of marginal cells of perichaetial leaves, seta colour, mature capsule shape, ornamentation of capsule, carriage of mature capsule on seta, shape of exothecial cells of the capsules, operculum type, type and shape of spores, spore ornamentation, peristome form, presence or absence of polysety and presence or absence of gemmae.

The morphological and anatomical parameters of the mature shoots representative of each of the species studied were described and documented with photomicrographs using an AmScope MT microscope camera version 3.0.0.1 attached to a light microscope.

\section{Anatomical studies}

Mature gametophytes from each moss taxon studied were washed with distilled water and cleared of dirt and other impurities. The chlorophyll content of the shoots was removed by soaking them in MacCartney bottles containing $10 \mathrm{ml}$ of Dimethyl-Sulphuroxide (DMSO) for 24 hours at $67^{\circ} \mathrm{C}$. The leaf cells and other anatomical parameters of the mature shoots were described and documented with photomicrographs using an AmScope MT microscope camera version 3.0.0.1 attached to a light microscope.

\section{Chromosome studies}

Mitotic chromosome preparations were made by pretreating young gametophytes of the moss species studied with $0.004 \mathrm{M}$ colchicine for 2 hours and then fixing in 1:1:1 ethanol:glacial acetic acid:chloroform for 48 hours. After this, the shoot apices were excised and squashed on glass slides and then stained with FLP Orcein ( 2 gm of orcein in $100 \mathrm{~cm}^{3}$ of solution containing equal parts of Formic acid, Lactic acid, Propionic acid and distilled water (Olorode, 1974) for 3 hours. Meiotic chromosome preparations were made by fixing freshly-harvested capsules at various stages of development in 1:3 acetic-ethanol for 48 hours. The capsules were then squashed on glass slides and stained with FLP orcein for 3 hours. Mitotic and meiotic cells with good spread representative of the species were documented by taking photomicrographs under phase contrast.

\section{Results and Discussions}

The mosses species that were investigated in this study are described below with respect to their general habit, habitat, qualitative and quantitative attributes of the gametophytes, sporophytes, leaf cells as well as chromosome as observed during the study.

\section{Morphological description of the moss species studied Hyophila crenulata}

Acrocarpous, saxicolous moss species (Fig. 1A) usually seen on concrete surfaces in shady areas; green when moist and when dry; perichaetial leaves (Fig.1F) mucronate, apex apiculate, margin denticulate, length $2.32 \pm 0.07 \mathrm{~mm}$, width $0.67 \pm 0.01 \mathrm{~mm}$, nerves extended to leaf apices; sporophytes (Fig. 2A) stegocarpous, erect; seta green when moist, straw brown when dry, seta length $90.50 \pm 3.37 \mathrm{~mm}$, seta width $0.11 \pm 0.01 \mathrm{~mm}$; mature capsules cylindrical, striate, operculate; operculum snouted; peristome (Fig. 2F) haplolepidous, spirally twisted; spores (Fig. $2 \mathrm{~K}$ ) psilate, sometimes circular, sometimes elliptic, sometimes tripantocolpate, sometimes trilete, sometimes polyzonocolpate, spores isodiametric, diameter up to 7.50 $\mu \mathrm{m}$; polysety seen.

Table 1. Sources of moss species studied

\begin{tabular}{|c|c|c|c|}
\hline Moss species & Collector/Source/Location & Habit & Habitat Description \\
\hline Hyophila crenulata & $\begin{array}{c}\text { Bolaji; Ile-Ife, } 7^{\circ} 31.45^{\prime} \mathrm{N} ; 4^{\circ} 31.46^{\prime} \mathrm{E} \text {, Nigeria } \\
7^{\circ} 31.48^{\prime} \mathrm{N} ; 4^{\circ} 31.46^{\prime} \mathrm{E} \text {, Nigeria }\end{array}$ & Acrocarpous & On concrete walls under tree shades \\
\hline Thuidium gratum & $\begin{array}{c}\text { Bolaji; Ile-Ife, } 7^{\circ} 31.25^{\prime} \mathrm{N} ; 4^{\circ} 31.31^{\prime} \mathrm{E} \text {, Nigeria } \\
7^{\circ} 31.45^{\prime} \mathrm{N} ; 4^{\circ} 31.48^{\prime} \mathrm{E} \text {, Nigeria } \\
7^{\circ} 31.25^{\prime} \mathrm{N} ; 4^{\circ} 31.31^{\prime} \mathrm{E} \text {, Nigeria } \\
7^{\circ} 31.46^{\prime} \mathrm{N} ; 4^{\circ} 31.48^{\prime} \mathrm{E} \text {, Nigeria }\end{array}$ & Pleurocarpous & $\begin{array}{l}\text { On tree bark around the base of tree (Milicia } \\
\text { excelsia, Dalbergi spp., Steculia tragacantha, } \\
\text { Funtumia elastica and Celtic zenkeri) }\end{array}$ \\
\hline Barbula lambarenensis & Bolaji; Ile-Ife, $7^{\circ} 31.46^{\prime} \mathrm{N} ; 4^{\circ} 31.48^{\prime} \mathrm{E}$, Nigeria & Acrocarpous & On concrete walls \\
\hline Stereophyllum nitense & $\begin{array}{c}\text { Bolaji; Ile-Ife, } 7^{\circ} 31.26^{\prime} \mathrm{N} ; 4^{\circ} 31.31^{\prime} \mathrm{E} \text {, Nigeria } \\
7^{\circ} 31.9^{\prime} \mathrm{N} ; 4^{\circ} 31.39^{\prime} \mathrm{E} \text {, Nigeria } \\
7^{\circ} 31.46^{\prime} \mathrm{N} ; 4^{\circ} 31.48^{\prime} \mathrm{E} \text {, Nigeria }\end{array}$ & Pleurocarpous & $\begin{array}{l}\text { On tree bark around the base of a tree (Acacia } \\
\text { spp., Funtumia elastic, Dalbergia spp. and } \\
\text { Lagerstomia indica) }\end{array}$ \\
\hline Bryum coronatum & Bolaji; Ile-Ife, $7^{\circ} 31.5^{\prime} \mathrm{N} ; 4^{\circ} 31.34^{\prime} \mathrm{E}$, Nigeria & Acrocarpous & $\begin{array}{l}\text { On concrete slabs along a pathway under } \\
\text { shade }\end{array}$ \\
\hline
\end{tabular}


406
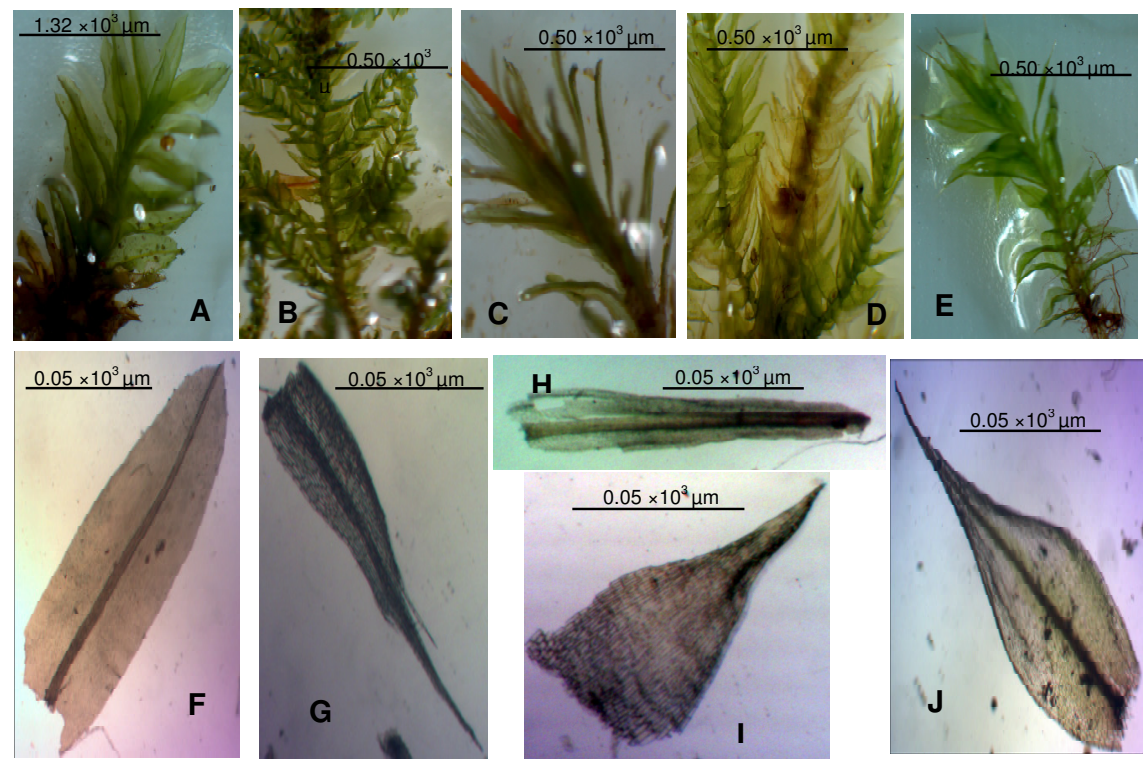

Fig. 1. Gametophytic morphological features of the moss species studied. A: Hyophila crenulata shoot; B: Thuidium gratum shoot; C: Barbula lambarenensis shoot; D: Stereophyllum nitense shoot E: Bryum coronatum shoot; F: Hyophila crenulata perichaetial leaf; G: Thuidium gratum perichaetial leaf; H: Barbula lambarenensis perichaetial leaf; I: Stereophyllum nitense perichaetial leaf; J: Bryum coronatum perichaetial leaf
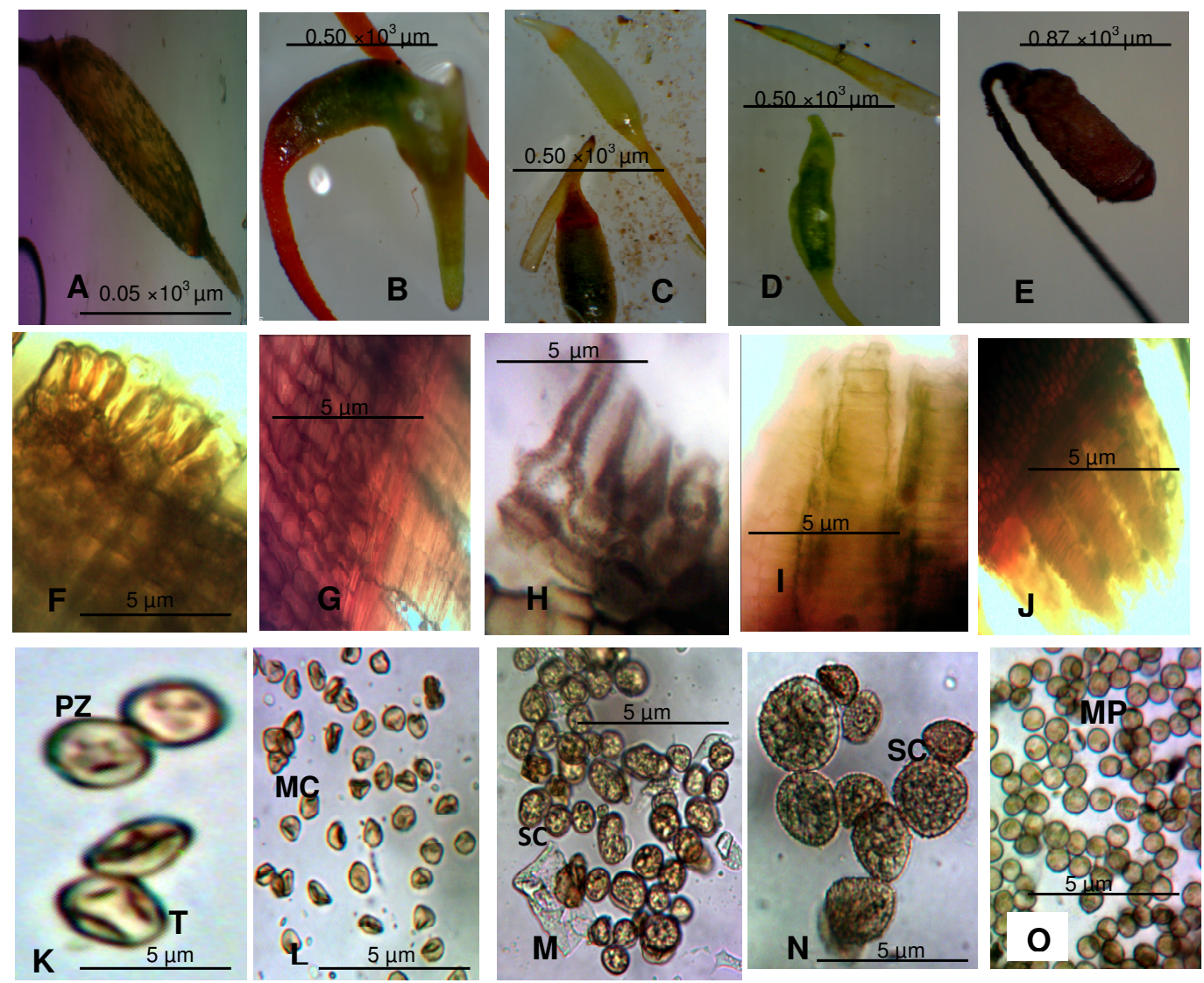

Fig. 2. Sporophytic morphological features of the moss species studied. A: Hyophila crenulata sporophyte; B: Thuidium gratum sporophyte; C: Barbula lambarenensis sporophyte; D: Stereophyllum nitense sporophyte; E: Bryum coronatum sporophyte; F: Hyophila crenulata peristome; G: Thuidium gratum peristome; H: Barbula lambarenensis peristome; I: Stereophyllum nitense peristome; J: Bryum coronatum peristome; K: Hyophila crenulata spore; L: Thuidium gratum spore; M: Barbula lambarenensis spore; N: Stereophyllum nitense spore; O: Bryum coronatum spore. Legends: PZ represents polyzonocolpate; T represents Trilete; $\mathrm{MC}$ represents monocolpate; SC represents scabrate; MP represents monoporate 
Table 2. Comparative morphology of the moss species studied

\begin{tabular}{|c|c|c|c|c|c|}
\hline Character & Hyophila crenulata & Thuidium gratum & Barbula lambarenensis & Stereophyllum nitense & Bryum coronatum \\
\hline Habit/ habitat & $\begin{array}{c}\text { Acrocarpous, } \\
\text { saxicolous, occurring } \\
\text { on concrete surfaces } \\
\text { in shady areas }\end{array}$ & $\begin{array}{l}\text { Pleurocarpous, corticolous, } \\
\text { occurring on tree barks } \\
\text { around the base of tree } \\
\text { trunks such as Milicia } \\
\text { excelsia, Dalbergi spp., } \\
\text { Sterculia tragacantha, } \\
\text { Funtumia elastica and Celtic } \\
\text { zenkeri }\end{array}$ & $\begin{array}{l}\text { Acrocarpous, terricolous, } \\
\text { occurring on concrete } \\
\text { surfaces in moist areas }\end{array}$ & $\begin{array}{c}\text { Pleurocarpous, } \\
\text { corticolous, occurring } \\
\text { around the base of trees } \\
\text { such as Funtumia elastica, } \\
\text { Acacia spp., Dalbergia } \\
\text { spp. and Lagerstomia } \\
\text { indica }\end{array}$ & $\begin{array}{c}\text { Acrocarpous, } \\
\text { terricolous, occurring } \\
\text { on concrete slabs along } \\
\text { pathway under shade }\end{array}$ \\
\hline Gametophyte & $\begin{array}{l}\text { Green when moist } \\
\text { and when dry; } \\
\text { perichaetial leaves } \\
\text { mucronate, length } \\
2.32 \pm 0.07 \mathrm{~mm}, \\
\text { width } 0.67 \pm 0.01 \\
\text { mm, apex apiculate, } \\
\text { margin denticulate, } \\
\text { nerves extended to } \\
\text { leaf apices }\end{array}$ & $\begin{array}{c}\text { Green when moist and } \\
\text { when dry; perichaetial leaves } \\
\text { lanceolate with excurrent } \\
\text { leaf point, length } 0.62 \pm \\
0.04 \mathrm{~mm} \text {, width } 0.13 \pm 0.01 \\
\text { mm, apex aristate, margin } \\
\text { entire, nerves excurrent }\end{array}$ & $\begin{array}{l}\text { Green when moist and } \\
\text { yellowish green when dry; } \\
\text { perichaetial leaves } \\
\text { ligulate, length } 1.57 \pm \\
0.04 \mathrm{~mm} \text {, width } 0.27 \pm \\
0.01 \mathrm{~mm} \text {, apices acute, } \\
\text { margins entire, nerves } \\
\text { extended up to leaf apices }\end{array}$ & $\begin{array}{l}\text { Green when moist, light } \\
\text { green when dry; } \\
\text { perichaetial leaves } \\
\text { cordate-triangular, length } \\
1.29 \pm 0.06 \mathrm{~mm} \text {, width } \\
0.42 \pm 0.03 \mathrm{~mm} \text {, apex } \\
\text { acute, margin entire, } \\
\text { nerves extended up to half } \\
\text { to three quarter length of } \\
\text { leaves }\end{array}$ & $\begin{array}{c}\text { Green when moist and } \\
\text { dark brown when dry; } \\
\text { perichaetial leaves } \\
\text { ovate with long } \\
\text { hyaline, length } 2.75 \pm \\
0.16 \mathrm{~mm} \text {, width } 0.53 \pm \\
0.03 \mathrm{~mm} \text {, apex aristate, } \\
\text { margin entire, nerves } \\
\text { extended to hyaline } \\
\text { apices }\end{array}$ \\
\hline Sporophytes & $\begin{array}{l}\text { Stegocarpous, erect; } \\
\text { seta green when } \\
\text { moist, straw brown } \\
\text { when dry, seta } \\
\text { length } 90.50 \pm 3.37 \\
\text { mm, seta width } 0.11 \\
\pm 0.01 \mathrm{~mm} \text {; mature } \\
\text { capsules cylindrical, } \\
\text { striate, operculate; } \\
\text { operculum snouted; } \\
\text { peristome } \\
\text { haplolepidous, } \\
\text { spirally twisted }\end{array}$ & $\begin{array}{l}\text { Stegocarpous, reflexed; seta } \\
\text { reddish brown when moist } \\
\text { and when dry, seta length } \\
11.10 \pm 0.38 \mathrm{~mm} \text {, seta } \\
\text { width } 0.15 \pm 0.01 \mathrm{~mm} \text {; } \\
\text { capsules cylindrical, smooth, } \\
\text { operculate; operculum } \\
\text { snouted; peristomes } \\
\text { haplolepidous, deeply } \\
\text { forked and in pairs }\end{array}$ & $\begin{array}{l}\text { Stegocarpous, erect; seta } \\
\text { green when moist, straw } \\
\text { brown when dry, seta } \\
\text { length } 4.08 \pm 0.25 \mathrm{~mm} \text {, } \\
\text { seta width } 0.11 \pm 0.00 \\
\text { mm; capsules cylindrical, } \\
\text { smooth and operculate; } \\
\text { operculum snouted } \\
\text { peristome haplolepidous, } \\
\text { deeply forked }\end{array}$ & $\begin{array}{l}\text { Stegocarpous, reflexed; } \\
\text { seta reddish brown when } \\
\text { moist and when dry, seta } \\
\text { length } 6.50 \pm 0.50 \mathrm{~mm} \text {, } \\
\text { width } 0.13 \pm 0.01 \mathrm{~mm} \text {; } \\
\text { capsule ovoid, furrowed, } \\
\text { operculate; operculum } \\
\text { snouted; peristomes } \\
\text { haplolepidous, deeply } \\
\text { forked }\end{array}$ & $\begin{array}{c}\text { Stegocarpous, } \\
\text { pendulous; seta } \\
\text { reddish brown when } \\
\text { moist and when dry, } \\
\text { seta length } 18.30 \pm \\
1.24 \mathrm{~mm}, \text { seta width } \\
0.17 \pm 0.01 \mathrm{~mm} ; \\
\text { mature capsules } \\
\text { cylindrical, furrowed, } \\
\text { operculate; operculum } \\
\text { blunt; peristome } \\
\text { haplolepidous, deeply } \\
\text { forked }\end{array}$ \\
\hline Spores & $\begin{array}{c}\text { Psilate, sometimes } \\
\text { circular, sometimes } \\
\text { elliptic, sometimes } \\
\text { tripantocolpate, } \\
\text { sometimes trilete, } \\
\text { sometimes } \\
\text { polyzonocolpate, } \\
\text { spores isodiametric, } \\
\text { diameter up to } 7.50 \\
\mu \mathrm{m}\end{array}$ & $\begin{array}{l}\text { Psilate, largely bean-shaped, } \\
\text { sometimes elliptic, } \\
\text { sometimes monocolpate, } \\
\text { sometimes syncolpate, } \\
\text { sometimes trilete, spore } \\
\text { diameter } 9.25 \pm 0.38 \mu \mathrm{m}\end{array}$ & $\begin{array}{l}\text { Scabrate, sometimes } \\
\text { circular, sometimes } \\
\text { elliptic, spore diameter } \\
12.50 \pm 0.75 \mu \mathrm{m}\end{array}$ & $\begin{array}{c}\text { Spores scabrate, } \\
\text { sometimes circular, } \\
\text { sometimes elliptic, spore } \\
\text { diameter } 17.25 \pm 1.02 \\
\mu \mathrm{m}\end{array}$ & $\begin{array}{c}\text { Psilate, circular, } \\
\text { sometimes } \\
\text { inaperturate, } \\
\text { sometimes } \\
\text { monoporate; spore } \\
\text { diameter } 9.75 \pm 0.25 \\
\mu \mathrm{m}\end{array}$ \\
\hline Gemmae & Not seen & Not seen & $\begin{array}{l}\text { Multicellular gammae } \\
\text { seen on leaf axils }\end{array}$ & Not seen & Not seen \\
\hline Polysety & Present & Not seen & Not seen & Not seen & Not seen \\
\hline
\end{tabular}

\section{Thuidium gratum}

Pleurocarpous, corticolous moss species (Fig. 1B) occurring on tree barks around the base of tree trunks such as Milicia excelsia, Dalbergi spp., Sterculia tragacantha, Funtumia elastica and Celtic zenkeri; gametophytes green when moist and when dry; perichaetial leaves (Fig. 1G) lanceolate with excurrent leaf point, apex aristate, margin entire, leaf length $0.62 \pm 0.04 \mathrm{~mm}$, leaf width $0.13 \pm 0.01$ $\mathrm{mm}$, nerves excurrent; sporophyte (Fig. $2 \mathrm{~B}$ ) stegocarpous, reflexed, seta reddish brown when moist and when dry, seta length $11.10 \pm 0.38 \mathrm{~mm}$, seta width $0.15 \pm 0.01 \mathrm{~mm}$; capsules cylindrical, smooth, operculate; operculum snouted; peristomes (Fig. 2G) haplolepidous, deeply forked and in pairs; spores (Fig. 2L) psilate, largely bean-shaped, sometimes elliptic, sometimes monocolpate, sometimes syncolpate, sometimes trilete, spore diameter $9.25 \pm 0.38$ $\mu \mathrm{m}$; polysety not seen.

\section{Barbula lambarenensis}

Acrocarpous, terricolous moss species (Fig. 1C) occurring on concrete surfaces in moist areas; gametophytes green when moist and yellowish green when dry; perichaetial leaves (Fig. $1 \mathrm{H}$ ) ligulate, apices acute, margins entire, leaf length $1.57 \pm 0.04 \mathrm{~mm}$, leaf width $0.27 \pm 0.01$ $\mathrm{mm}$, nerves extended up to leaf apices; sporophyte (Fig. 2C) stegocarpous, erect; seta green when moist, straw brown when dry, seta length $4.08 \pm 0.25 \mathrm{~mm}$, seta width $0.11 \pm 0.00 \mathrm{~mm}$; capsules cylindrical, smooth and operculate; operculum snouted; peristome (Fig. $2 \mathrm{H}$ ) haplolepidous, deeply forked; spores (Fig. 2M) scabrate, sometimes circular, sometimes elliptic, spore diameter 12.50 $\pm 0.75 \mu \mathrm{m}$; polysety not seen; gemmae seen on leaf axils.

\section{Stereophyllum nitense}

Pleurocarpous, corticolous moss species (Fig. 1D) seen 
408

around the base of trees such as Funtumia elastica, Acacia spp., Dalbergia spp. and Lagerstomia indica; gametophytes green when moist, light green when dry; perichaetial leaves (Fig. 1I) cordate-triangular, apex acute, margin entire, length $1.29 \pm 0.06 \mathrm{~mm}$, width $0.42 \pm 0.03 \mathrm{~mm}$, nerves extended up to half to three quarter length of leaves; sporophytes (Fig. 2D) stegocarpous, reflexed; seta reddish brown when moist and when dry, seta length $6.50 \pm 0.50$ $\mathrm{mm}$, width $0.13 \pm 0.01 \mathrm{~mm}$; capsule ovoid, furrowed, operculate; operculum snouted; peristomes (Fig. 2I) haplolepidous, deeply forked; spores (Fig. 2N) scabrate, sometimes circular, sometimes elliptic, spores diameter $17.25 \pm 1.02 \mu \mathrm{m}$; polysety not seen.

\section{Bryum coronatum}

Acrocarpous, terricolous moss species (Fig. 1E), seen on soil surfaces of concrete slabs along pavements and pathways under moderate shade and at times around openings of drainage; gametophytes green when moist and dark brown when dry; perichaetial leaves (Fig.1J) ovate with long hyaline, length $2.75 \pm 0.16 \mathrm{~mm}$, width $0.53 \pm 0.03 \mathrm{~mm}$, apex aristate, margin entire, nerves extended to hyaline apices; sporophyte (Fig. 2E) stegocarpous, pendulous; seta reddish brown when moist and when dry, seta length 18.30 $\pm 1.24 \mathrm{~mm}$, seta width $0.17 \pm 0.01 \mathrm{~mm}$; mature capsules cylindrical, furrowed, operculate; operculum blunt; peristome (Fig. 2J) haplolepidous, deeply forked; spores (Fig. 2O) psilate, circular, sometimes inaperturate, sometimes monoporate; spore diameter $9.75 \pm 0.25 \mu \mathrm{m}$; gemmae not seen.

\section{Leaf anatomy of the moss species studied \\ Hyophila crenulata}

Lamina cells at upper region (Fig. 3A) of perichaetial leaves largely irregular, lamina cell length $8.29 \pm 0.25 \mu \mathrm{m}$, lamina cell width; lamina cells at middle portion (Fig. 3F) of leaves largely polygonal, lamina cell length $8.64 \pm 0.36 \mu \mathrm{m}$, lamina cell width $7.43 \pm 0.30 \mu \mathrm{m}$; lamina cells at leaf base (Fig. 3K) largely irregular, lamina cell length $28.50 \pm 1.19$ $\mu \mathrm{m}$, lamina cell width $11.36 \pm 0.40 \mu \mathrm{m}$; exothecial cells of capsules (Fig. 3P) irregular, anticlinal walls slightly wavy, cross walls perpendicular, sometimes oblique, exothecial cell length $0.41 \pm 0.03 \mu \mathrm{m}$, exothecial cell width $14.25 \pm 1.75$ $\mu \mathrm{m}$.

\section{Thuidium gratum}

Lamina cells at upper region of perichaetial leaves (Fig. 3B) irregular, at least 2 - 3 times longer than broad, cross walls largely oblique, occasionally perpendicular, anticlinal walls wavy, lamina cell length $37.79 \pm 2.44 \mu \mathrm{m}$, lamina cell width $7.57 \pm 0.16 \mu \mathrm{m}$; lamina cells at middle portion of leaves (Fig. 3G) largely irregular, sometimes rectangular, 2 4 times longer than broad, cross walls largely oblique, sometimes perpendicular, anticlinal walls wavy, lamina cell length $57.36 \pm 2.92 \mu \mathrm{m}$, lamina cell width $11.29 \pm 0.36 \mu \mathrm{m}$; lamina cells at the leaf base (Fig. 3L) largely irregular, lamina cell length $40.50 \pm 1.96 \mu \mathrm{m}$, lamina cell width $13.07 \pm 0.59$ $\mu \mathrm{m}$; exothecial cells of capsules (Fig. 3Q) largely irregular, sometimes polygonal, anticlinal walls wavy, exothecial cell length $0.38 \pm 0.04 \mu \mathrm{m}$, exothecial cell width $22.25 \pm 1.32$ $\mu \mathrm{m}$.

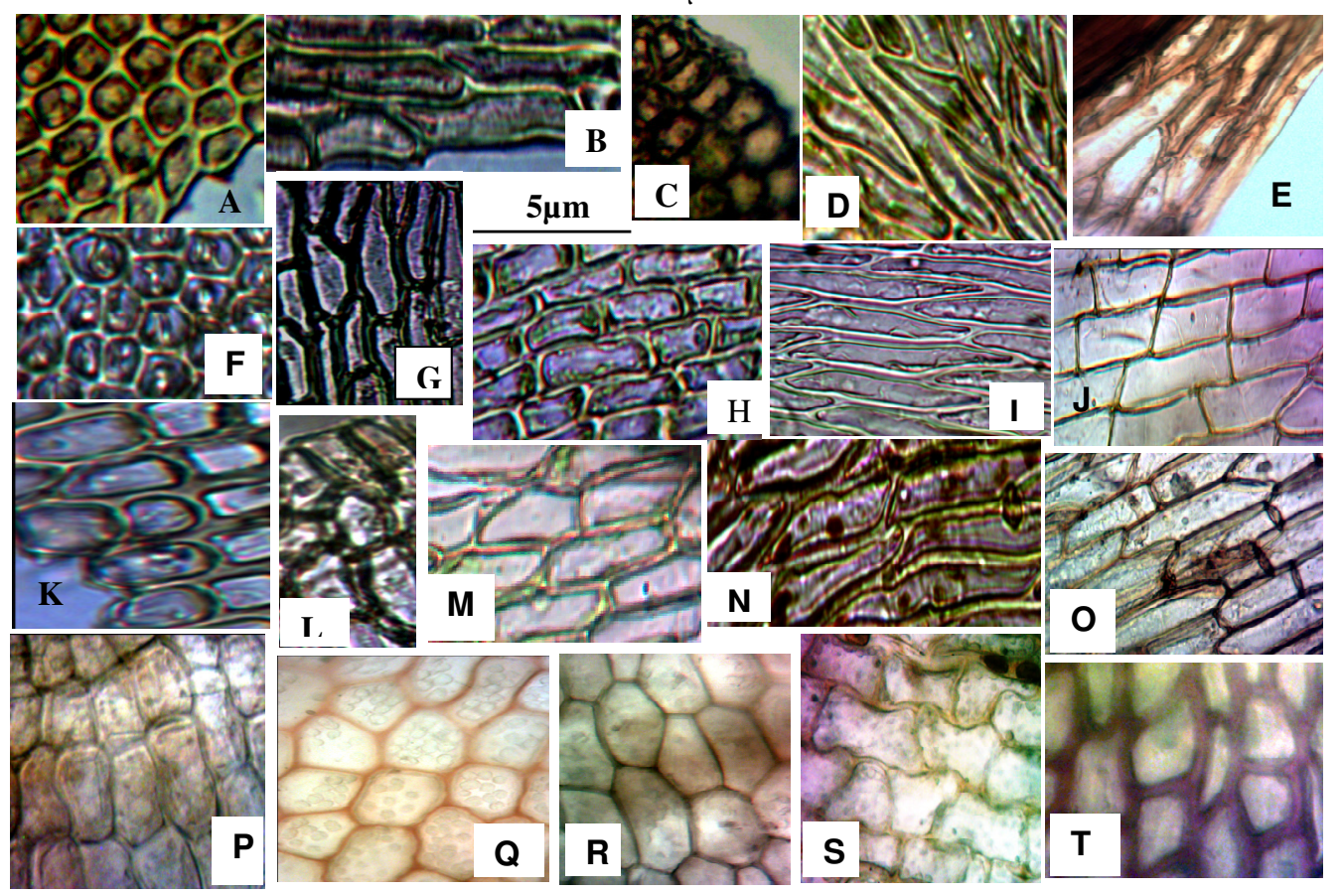

Fig. 3. Anatomical features of the Lamina cells and Exothecial cells of the moss species studied. A: Upper region of Hyophila crenulata leaf; B: Upper region of Thuidium gratum leaf; C: Upper region of Barbula lambarenensis leaf; D: Upper region of Stereophyllum nitense leaf; E: Upper region of Bryum coronatum leaf; F: Mid-region of Hyophila crenulata leaf; G: Mid-region of Thuidium gratum leaf; H: Mid-region of Barbula lambarenensis leaf; I: Mid-region of Stereophyllum nitense leaf; J: Mid-region of Bryum coronatum leaf; K: Basal region of Hyophila crenulata leaf; L: Basal region of Thuidium gratum leaf; M: Basal region of Barbula lambarenensis leaf; N: Basal region of Stereophyllum nitense leaf; O: Basal region of Bryum coronatum capsule; P: Exothecial cells of Hyophila crenulata capsule; Q: Exothecial cells of Thuidium gratum capsule; R: Exothecial cells of Barbula lambarenensis capsule; S: Exothecial cells of Stereophyllum nitense capsule; T: Exothecial cells of Bryum coronatum capsule 
Table 3. Comparative anatomy of the leaf and exothecial cells of capsules of the moss species studied

\begin{tabular}{|c|c|c|c|c|c|}
\hline & Hyophila crenulata & Thuidium gratum & Barbula lambarenensis & Stereophyllum nitense & Bryum coronatum \\
\hline $\begin{array}{l}\text { Upper } \\
\text { Region of } \\
\text { Leaf }\end{array}$ & $\begin{array}{l}\text { Lamina cells largely } \\
\text { irregular, lamina cell } \\
\text { length } 8.29 \pm 0.25 \mu \mathrm{m}, \\
\text { lamina cell width } 7.36 \\
\pm 0.10 \mu \mathrm{m}\end{array}$ & $\begin{array}{l}\text { Lamina cells irregular, at } \\
\text { least } 2 \text { - } 3 \text { times longer than } \\
\text { broad, cross walls largely } \\
\text { oblique, occasionally } \\
\text { perpendicular, anticlinal } \\
\text { walls straight, lamina cell } \\
\text { length } 37.79 \pm 2.44 \mu \mathrm{m} \text {, } \\
\text { lamina cell width } 7.57 \pm \\
0.16 \mu \mathrm{m} \text {; }\end{array}$ & $\begin{array}{l}\text { Lamina cells irregular, } \\
\text { lamina cell length } 8.21 \pm \\
0.36 \mu \mathrm{m} \text {, lamina cell width } \\
5.79 \pm 0.20 \mu \mathrm{m}\end{array}$ & $\begin{array}{l}\text { Lamina cells irregular, } 2 \\
-3 \text { times longer than } \\
\text { broad, cross walls } \\
\text { oblique, anticlinal walls } \\
\text { wavy, lamina cell length } \\
43.29 \pm 2.52 \mu \mathrm{m}, \\
\text { lamina cell width } 6.57 \\
\pm 0.27\end{array}$ & $\begin{array}{l}\text { Lamina cells largely } \\
\text { irregular, } 3-4 \text { times longer } \\
\text { than broad, cross wall } \\
\text { oblique, anticlinal walls } \\
\text { straight, lamina cell length } \\
56.86 \pm 2.56 \mu \mathrm{m} \text {, lamina } \\
\text { cell width } 12.50 \pm 1.04 \mu \mathrm{m} \text {; }\end{array}$ \\
\hline $\begin{array}{l}\text { Middle } \\
\text { Portion of } \\
\text { Leaf }\end{array}$ & $\begin{array}{c}\text { lamina cells at middle } \\
\text { portion of leaves } \\
\text { largely polygonal, } \\
\text { lamina cell length } 8.64 \\
\pm 0.36 \mu \mathrm{m} \text {, lamina cell } \\
\text { width } 7.43 \pm 0.30 \mu \mathrm{m}\end{array}$ & $\begin{array}{c}\text { lamina cells largely } \\
\text { irregular, sometimes } \\
\text { rectangular, } 2 \text { - } 4 \text { times } \\
\text { longer than broad, cross } \\
\text { walls largely oblique, } \\
\text { sometimes perpendicular, } \\
\text { anticlinal walls slightly } \\
\text { wavy, lamina cell length } \\
57.36 \pm 2.92 \mu \mathrm{m} \text {, lamina } \\
\text { cell width } 11.29 \pm 0.36 \mu \mathrm{m} \text {; }\end{array}$ & $\begin{array}{c}\text { lamina cells irregular, } \\
\text { cross walls sometimes } \\
\text { oblique, sometimes } \\
\text { perpendicular anticlinal } \\
\text { walls wavy, lamina cell } \\
\text { length } 25.57 \pm 1.91 \mu \mathrm{m} \text {, } \\
\text { lamina cell width } 5.79 \pm \\
0.25 \mu \mathrm{m} \text {; }\end{array}$ & $\begin{array}{l}\text { lamina cells irregular, } 2 \\
\text { - } 4 \text { times longer than } \\
\text { broad, cross walls } \\
\text { oblique, anticlinal walls } \\
\text { wavy, lamina cell length } \\
75.79 \pm 2.71 \mu \mathrm{m}, \\
\text { lamina cell width } 8.14 \\
\pm 0.28 \mu \mathrm{m}\end{array}$ & $\begin{array}{l}\text { lamina cells largely irregular, } \\
2-4 \text { times longer than } \\
\text { broad, cross walls oblique, } \\
\text { anticlinal walls wavy, } \\
\text { lamina cell length } 70.79 \pm \\
2.57 \mu \mathrm{m} \text {, lamina cell width } \\
15.50 \pm 0.71 \mu \mathrm{m} ;\end{array}$ \\
\hline Leaf Base & $\begin{array}{c}\text { lamina cells at leaf base } \\
\text { largely irregular, } \\
\text { lamina cell length } \\
28.50 \pm 1.19 \mu \mathrm{m}, \\
\text { lamina cell width } \\
11.36 \pm 0.40 \mu \mathrm{m}\end{array}$ & $\begin{array}{c}\text { lamina cells largely } \\
\text { irregular, lamina cell length } \\
40.50 \pm 1.96 \mu \mathrm{m} \text {, lamina } \\
\text { cell width } 13.07 \pm 0.59 \mu \mathrm{m} \text {; }\end{array}$ & $\begin{array}{c}\text { lamina cells largely } \\
\text { irregular, } 2-3 \text { times } \\
\text { longer than broad, cross } \\
\text { walls sometimes oblique, } \\
\text { sometimes perpendicular, } \\
\text { anticlinal walls wavy, } \\
\text { lamina cell length } 33.79 \\
\pm 2.33 \mu \mathrm{m} \text {, lamina cell } \\
\text { width } 8.79 \pm 0.31 \mu \mathrm{m}\end{array}$ & $\begin{array}{c}\text { lamina cells largely } \\
\text { irregular, } 3 \text { - } 5 \text { times } \\
\text { longer than broad, cross } \\
\text { walls largely oblique, } \\
\text { anticlinal walls wavy, } \\
\text { lamina cell length } 52.07 \\
\pm 2.84 \mu \mathrm{m} \text {, lamina cell } \\
\text { width } 9.36 \mu \mathrm{m} \pm 0.37 \\
\mu \mathrm{m} ;\end{array}$ & $\begin{array}{c}\text { lamina cells largely } \\
\text { polygonal, sometimes } \\
\text { irregular, } 2-4 \text { times longer } \\
\text { than broad, cross walls } \\
\text { oblique, sometimes } \\
\text { perpendicular, anticlinal } \\
\text { walls straight, lamina cell } \\
\text { length } 56.07 \pm 2.38 \mu \mathrm{m} \text {, } \\
\text { lamina cell width } 17.57 \pm \\
0.78 \mu \mathrm{m} ;\end{array}$ \\
\hline $\begin{array}{c}\text { Exothecial } \\
\text { Cells of } \\
\text { Capsule }\end{array}$ & $\begin{array}{c}\text { Irregular, anticlinal } \\
\text { walls slightly wavy, } \\
\text { cross walls } \\
\text { perpendicular, } \\
\text { sometimes oblique, } \\
\text { exothecial cell length } \\
0.41 \pm 0.03 \mu \mathrm{m}, \\
\text { exothecial cell width } \\
14.25 \pm 1.75 \mu \mathrm{m}\end{array}$ & $\begin{array}{c}\text { Largely irregular, } \\
\text { sometimes polygonal, } \\
\text { anticlinal walls wavy, } \\
\text { exothecial cell length } 0.38 \\
\pm 0.04 \mu \mathrm{m} \text {, exothecial cell } \\
\text { width } 22.25 \pm 1.32 \mu \mathrm{m}\end{array}$ & $\begin{array}{c}\text { Largely polygonal, } \\
\text { sometimes irregular, } \\
\text { exothetial cell length } 0.33 \\
\pm 0.03 \mu \mathrm{m} \text {, exothecial cell } \\
\text { width } 20.75 \pm 1.49 \mu \mathrm{m}\end{array}$ & $\begin{array}{l}\text { Exothecial cells of } \\
\text { capsules largely } \\
\text { irregular, sometimes } \\
\text { rectangular, anticlinal } \\
\text { walls wavy, exothecial } \\
\text { cell length } 0.44 \pm 0.03 \\
\mu \mathrm{m} \text {, exothecial cell } \\
\text { width } 20.50 \pm 1.62 \mu \mathrm{m} \text {. }\end{array}$ & $\begin{array}{l}\text { Largely irregular, sometimes } \\
\text { rectangular, anticlinal walls } \\
\text { slightly wavy, cross walls } \\
\text { largely oblique, exothecial } \\
\text { cell length } 32.00 \pm 2.38 \mu \mathrm{m} \text {, } \\
\text { exothecial cell width } 0.63 \pm \\
0.03 \mu \mathrm{m}\end{array}$ \\
\hline
\end{tabular}

\section{Barbula lambarenensis}

Lamina cells at upper region of perichaetial leaves (Fig. 3C) irregular, lamina cell length $8.21 \pm 0.36 \mu \mathrm{m}$, lamina cell width $5.79 \pm 0.20 \mu \mathrm{m}$; lamina cells at middle portion of leaves (Fig. $3 \mathrm{H}$ ) largely rectangular, cross walls sometimes perpendicular, sometimes oblique; anticlinal walls wavy, lamina cell length $25.57 \pm 1.91 \mu \mathrm{m}$, lamina cell width $5.79 \pm$ $0.25 \mu \mathrm{m}$; lamina cells at leaf base (Fig. 3M) irregular, $2-3$ times longer than broad, cross walls perpendicular, sometimes oblique, anticlinal walls wavy, lamina cell length $33.79 \pm 2.33 \mu \mathrm{m}$, lamina cell width $8.79 \pm 0.31 \mu \mathrm{m}$; exothecial cells of capsules (Fig. 3R) largely polygonal, sometimes irregular, exothetial cell length $0.33 \pm 0.03 \mu \mathrm{m}$, exothecial cell width $20.75 \pm 1.49 \mu \mathrm{m}$.

\section{Stereophyllum nitense}

Lamina cells at upper region of perichaetial leaves (Fig. 3D) irregular, $2-3$ times longer than broad, cross walls oblique, anticlinal walls wavy, lamina cell length $43.29 \pm$ $2.52 \mu \mathrm{m}$, lamina cell width $6.57 \pm 0.27 \mu \mathrm{m}$; lamina cells at middle portion of leaves (Fig. 3I) irregular, 2 - 4 times longer than broad, cross walls oblique, anticlinal walls wavy, lamina cell length $75.79 \pm 2.71 \mu \mathrm{m}$, lamina cell width $8.14 \pm 0.28$ $\mu \mathrm{m}$; lamina cells at leaf base (Fig. $3 \mathrm{~N}$ ) largely irregular, 3-5 times longer than broad, cross walls largely oblique, anticlinal walls wavy, lamina cell length $52.07 \pm 2.84 \mu \mathrm{m}$, lamina cell width $9.36 \mu \mathrm{m} \pm 0.37 \mu \mathrm{m}$; lamina cells at leaf margin of perichaetial leaves irregular, sometimes rectangular; exothecial cells of capsules (Fig. 3S) largely irregular, sometimes rectangular, anticlinal walls wavy, exothecial cell length $0.44 \pm 0.03 \mu \mathrm{m}$, exothecial cell width $20.50 \pm 1.62 \mu \mathrm{m}$.

\section{Bryum coronatum}

Lamina cells at upper region of perichaetial leaves (Fig. 3E) largely irregular, 3 - 4 times longer than broad, cross wall oblique, anticlinal slightly wavy, lamina cell length $56.86 \pm$ $2.56 \mu \mathrm{m}$, lamina cell width $12.50 \pm 1.04 \mu \mathrm{m}$; lamina cells at middle portion of leaves (Fig. 3J) largely irregular, 2 - 4 times longer than broad, lamina cell length $70.79 \pm 2.57 \mu \mathrm{m}$, lamina cell width $15.50 \pm 0.71 \mu \mathrm{m}$; lamina cells at leaf base (Fig. 3O) largely polygonal, sometimes irregular, 2 - 4 times longer than broad, cross walls oblique, sometimes perpendicular, anticlinal walls slightly wavy, lamina cell length $56.07 \pm 2.38 \mu \mathrm{m}$, lamina cell width $17.57 \pm 0.78 \mu \mathrm{m}$; exothecial cells of capsules (Fig. 3T) largely irregular, sometimes rectangular, anticlinal walls slightly wavy, cross walls largely oblique, length $32.00 \pm 2.38 \mu \mathrm{m}$, exothecial cell width $0.63 \pm 0.03 \mu \mathrm{m}$. 
410

\section{Chromosomes of the moss species studied}

The Hyophila crenulata studied showed a chromosome number of $n=4$. The metaphase I cells (Fig. 4A) showed the occurrence of four bivalent chromosomes (4II) with callous walls surrounding the cells. The Thuidium gratum studied showed a chromosome number of $n=12(10+$ $2 \mathrm{~m}$ ). The metaphase I (Fig. $4 \mathrm{~B}$ ) revealed the presence of two chromosomes which were usually smaller and lightly stained (arrowed), but always seen as part of the chromosome complement. These were regarded as m-chromosomes. Barbula lambarenensis studied showed a chromosome number of $2 n=6$ (Fig. 4C). Two of the chromosome complements were conspicuously large. The Stereophyllum nitense studied showed a chromosome number of $2 n=18$ chromosomes (Fig. 3D). The Bryum coranatum studied showed a chromosome number of $n=10$ (Fig. 4E). The chromosomes of the five moss species studied were usually sticky and tend to always clump together at metaphase I. This was more notable in Stereophyllum nitense, Thuidium gratum and Bryum coranatum.
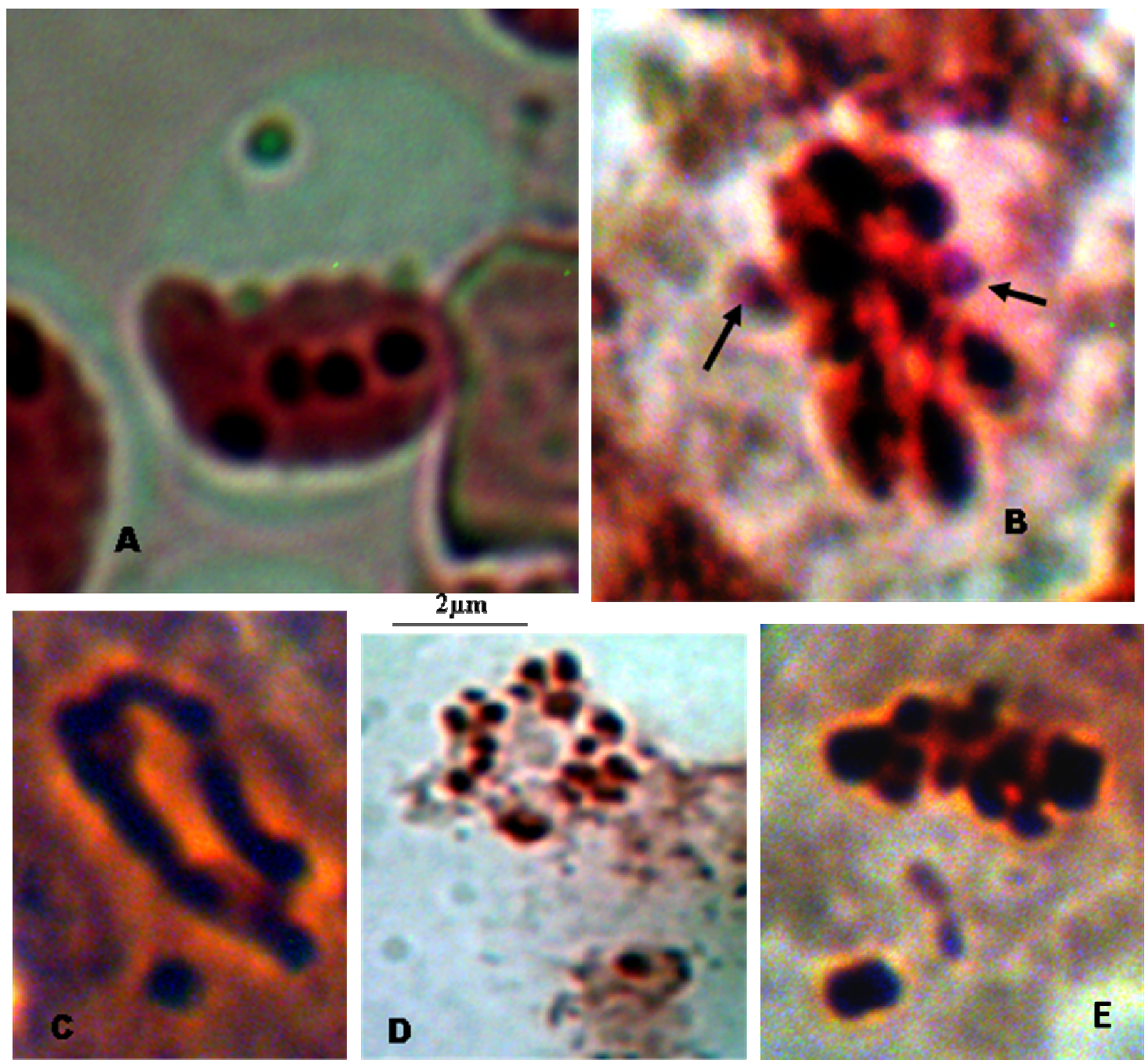

Fig. 4. Chromosomes of the moss species studied. A: Metaphase I of Hyophila crenulata showing 4 bivalent (4II) chromosomes (i.e. $n=4)$; B: Metaphase I of Thuidium gratum showing $n=12(10+2 \mathrm{~m})$ chromosomes (m-chromosomes arrowed); C: Mitotic metaphase of Barbula lambarenensis showing $2 n=6$ chromosomes; D: Mitotic metaphase cell of Stereophyllum nitense showing $2 n=18$ chromosomes; E: Metaphase I cell of Bryum coronatum showing $n=10$ chromosomes 
cell width increased from the apex to the basal region in all the species studied.

At the apices and upper regions of the leaves, the lamina cells were generally irregular in all the species studied, but their sizes and arrangement within the leaves varied (Fig. 3 and Table 3). While the lamina cells were much longer than broad in Thuidium gratum (37.79 $\pm 2.44 \mu \mathrm{m}$ long, $7.57 \pm$ $0.16 \mu \mathrm{m}$ broad), Stereophyllum nitens (43.29 $\pm 2.52 \mu \mathrm{m}$ long, $6.57 \pm 0.27$ broad) and Bryum coronatum (56.86 \pm $2.56 \mu \mathrm{m}$ long, $12.50 \pm 1.04 \mu \mathrm{m}$ broad), they were shorter in Hyophila crenulata $(8.29 \pm 0.25 \mu \mathrm{m}$ long, $7.36 \pm 0.10 \mu \mathrm{m}$ broad) and Barbula lambarenensis (8.21 $\pm 0.36 \mu \mathrm{m}$ long, $5.79 \pm 0.20 \mu \mathrm{m}$ broad).

At the middle portion of the leaves the leaf cells were irregular in all except in Hyophila crenulata where the lamina cells were polygonal. The sizes of the leaf cells, their shapes and arrangement also varied in this portion of the leaves. While they were much longer than broad in Thuidium gratum $(57.36 \pm 2.92 \mu \mathrm{m}$ long, $11.29 \pm 0.36 \mu \mathrm{m}$ broad), Barbula lambarenensis ( $25.57 \pm 1.91 \mu \mathrm{m}$ long, 5.79 $\pm 0.25 \mu \mathrm{m}$ broad), Stereophyllum nitens (75.79 $\pm 2.71 \mu \mathrm{m}$ long, $8.14 \pm 0.28$ broad) and Bryum coronatum (70.79 \pm $2.57 \mu \mathrm{m}$ long, $15.50 \pm 0.71 \mu \mathrm{m}$ broad), they were shorter in Hyophila crenulata $(8.64 \pm 0.36 \mu \mathrm{m}$ long, $7.43 \pm 0.30 \mu \mathrm{m}$ broad).

At the basal region of the leaves the lamina cells were all irregular though the shapes, lamina cell sizes and arrangement differed from one species to the other. The lamina cell length was much longer than broad at the basal region in all the species studied.

The exothecial cells of the capsules of the species studied also varied in shape, sizes and arrangement (Fig. 3 and Table 3 ) and are diagnostic for the species studied. Although the shapes were irregular in all the species studied, the shapes, sizes and arrangement varied across the species.

The anatomical characters of the leaves and capsules of the moss species studied were diagnostic hence, can be taxonomically employed to delimit the species from each other. Anatomical parameters of different plant parts have been used as aids in the taxonomic recognition of species (Kathiresan et al., 2011). Schofield (1985) noted that cell shapes and arrangement within leaves of moss species usually differ remarkably and they provide some of the most reliable characters that could be used to distinguish them.

The sporophytic features of the moss species studied also varied distinctly across the species studied. Though they were all stegocarpous, the capsule shapes, capsule carriage, operculum type, peristome type and spore varied distinctly across the species studied. While the capsules were cylindrical and smooth in Thuidium gratum and Barbula lambarenensis, they were cylindrical and striate in Hyophila crenulata, cylindrical and furrowed in Bryum coronatum, ovoid and furrowed in Stereophyllum nitense. The capsule carriage was erect in Hyophila crenulata and Barbula lambarenensis while they were reflexed in Thuidium gratum and Stereophyllum nitense and pendulous in Bryum coronatum. The operculum was snouted in all the species studied except in Bryum coronatum where they were blunt. The peristomes were spirally twisted in Hyophila crenulata, deeply forked and paired in Thuidium gratum, deeply forked and unpaired in Barbula lambarenensis, Stereophyllum nitense and Bryum coronatum though their shapes varied.

The spores were psilate (smooth) in Hyophila crenulata, Thuidium gratum and Bryum coronatum, while they were scabrate in Barbula lambarenensis and Stereophyllum nitense. They were circular sometimes elliptic in Hyophila crenulata, Barbula lambarenensis and Stereophyllum nitense, largely circular in Bryum coronatum and largely bean-shaped though sometimes elliptic in Thuidium gratum. The spores of the various species studied were quite distinct with respect to their spore ornamentations and aperture. The spores were tripantocolpate, sometimes trilete, sometimes polyzonocolpate in Hyophila crenulata; monocolpate, sometimes syncolpate, sometimes trilete in Thuidium gratum; inaperturate in Barbula lambarenensis and Stereophyllum nitense and Bryum coronatum. The spores were generally small in size and quite numerous in all the species studied. Spore diameter was largest in Stereophyllum nitense $(17.25 \pm 1.02 \mu \mathrm{m})$ and least in Hyophila crenulata $(7.50 \pm 0.00 \mu \mathrm{m})$, while in Barbula lambarenensis, Bryum coronatum and Thuidium gratum they were $12.50 \pm 0.75$ $\mu \mathrm{m}, 9.75 \pm 0.25 \mu \mathrm{m}$ and $9.25 \pm 0.38 \mu \mathrm{m}$ respectively.

Capsule carriage and texture, peristome form, spore ornamentation of mature sporophytes are reliable characters that can be useful in delimiting the species studied since they are usually genetically determined.

The occurrence of gemmae noticed on only Barbula lambarenensis (Table 2) out of the five species studied, is an indication of an asexual strategy of propagation and spread; while the occurrence of polysety in Hyophila crenulata is an indication of strategy for population spread by sexual means. According to Schofield (1985), gemmae serve as diaspores that engage in vegetative reproduction and are important in the expansion of local populations. Oyesiku (2012) speculated that the mechanism behind polysety is not fully known, but may be because of simultaneous fertilization of two or more individuals of archegonia, aided by sugary exudates from the mature archegonia.

The occurrence of sporophytes that were stegocarpous and operculate with spores that were relatively small in size and numerous, in all the species studied, suggest the likelihood of long distance dispersal of their spores and thus possibility of their occurrence over a wide range of geographical location provided the habitat conditions are conducive with respect to moisture, $\mathrm{pH}$ and other environmental conditions.

The chromosome numbers of Hyophila crenulata, Thuidium gratum, Barbula lambarenensis, Stereophyllum nitense and Bryum coronatum from Nigeria are revealed for the first time in this study. Hyophila crenulata has a chromosome count of $n=4$; Thuidium gratum has a chromosome count of $n=12(10+2 \mathrm{~m})$; Barbula lambarenensis has a chromosome count of $n=3$ (i.e. $2 n=$ 6); while Stereophyllum nitense has a chromosome count of $n=9(2 n=18)$. There is no known previous record of chromosome numbers of Hyophila crenulata, Thuidium gratum, Barbula lambarenensis and Stereophyllum nitense being reported in this study. However, there are known records of Bryum coronatum from other parts of the world. 
412

Kumar et al. (1988) reported the chromosome number of Bryum coronatum as $n=10$.

The occurrence of chromosomes which were usually sticky and tend to always clump together at Metaphase I is also noteworthy and being reported in this study. Its notable occurrence in Stereophyllum nitense, Thuidium gratum and Bryum coronatum than in the other species studied, could be because of the relatively larger chromosome numbers in their complements (i.e. $n=9, n=12$ and $n=10$ respectively). Stickiness of chromosomes was also reported in moss species in the family Brachytheciaceae by McAdam (1982).

In this study, the presence of m-chromosomes was found only in Thuidium gratum. Reports on the presence of $\mathrm{m}$-chromosomes and accessory chromosomes in bryophytes have shown that in both cases, the chromosomes are smallest in size compared to the other members of the complement. However while m-chromosomes were always present in the complement, accessory chromosomes may or may not be present (Ramsay, 1964, 1969; Snider, 1973; Muntung, 1974). Also, light-staining and precocious segregation are diagnostic of $\mathrm{m}$-chromosomes while accessory chromosomes usually present mitotic/meiotic irregularities resulting in numerical variation in chromosomes of species or populations (Ramsay, 1964, 1969; Snider, 1973; Muntung, 1974).

\section{Conclusions}

Some details of the morphological and anatomical attributes as well as chromosome numbers of Hyophila crenulata, Thuidium gratum, Barbula lambarenensis, Stereophyllum nitense and Bryum coronatum from Nigeria are being reported for the first time in this study and could be very useful in the identification and taxonomic delimitation of these species. However, more detailed morphometric and molecular studies are required to establish their taxonomic status and evolutionary relationship.

\section{References}

Adebiyi AO, Oyedeji AA, Chukwendu EE, Fatoke OA (2012). Phytochemical screening of two tropical moss plants: Thuidium gratum P. Beauv. and Barbula indica Brid. Grown in Southwestern ecological zone of Nigeria. American Journal of Analytical Chemistry 3:836-839.

Adebiyi AO, Tedela PO, Oyedeji AA, Agunbiade RO, Bawo DDS (2013). Evaluation of the efficacy of moss plants: Thuidium gratum P. Beauv. and Barbula indica Brid. grown in Southwestern ecological zone of Nigeria on the survival of Callosobruchus maculates (F.) Coleoptera: Bruchidae. American Journal of Food Nutrition 3(1):9-12.

Aline CM, Rosane L, Jucara B, Alois S, Ronaldo W (2012). Mosses as indicators of atmospheric metal deposition in an industrial area of Southern Brazil. Acta Botanica Brasilica 26(3):553-558.

AndeAT, WahediJA, Fatoba PO (2010). Biocidal activities of some tropical moss extracts against maize stem borers. Ethnobotanical Leaflets 14:479490.

Batagarawa SM, Lawal AO (2010). Determination of some heavy metals levels in Funaria hygrometrica in Dutsinma town of Katsina State, Nigeria. Journal of AppliedEnvironmental Management 14(3):65-68.

Bobade RG, Bakar PS, Saiful AM, Khobragade CN (2008). In vitro screening of bryophytes for antimicrobial activity. Journal of Medicinal Plants Research 7(4):23-28.

Buck WR, Goffinet B (2000). Morphology and classification of mosses. In: Shaw AJ, Goffinet B (Eds). Bryophyte Biology. Cambridge: Cambridge University Press.

Davidson AJ, Harborne JB, Longton RE(1990). The acceptability of mosses as food for generalist herbivores slugs in the arionidea. Botanical Journal of the Linnaean Society 104:99-113.

Dickson JH (1997). The moss from the tyrolean iceman's colon. Journal of Bryology 19:449-451.

Dulger B, Yayintas OT, Gonuz A (2005). Antimicrobial activity of some mosses from Turkey. Fitoterapia 76:730-732

Egunyomi A (1979). A checklist of mosses in Southwestern Nigeria. Nigerian Journal of Science 13:483-492.

Egunyomi A (1980). Mosses from Nigeria III: New records from the Middle-Belt area. Journal of Hattori Botanical Laboratory 48:187-193.

Egunyomi A (1981). Checklist of mosses in Northern and Southern Nigeria. Nigerian Journal of Forestry 11(2):63-67.

Egunyomi A (1984). New records for the moss flora of Nigeria. Bryologist 87(4):369-370.

Egunyomi A, Vital DM (1984). Comparative studies in the bryoflora of the Nigerian Savanna and the Brazillian cerrado. Revista Brasileira de Botânica 7(2):129-136.

Ekpo BO, Uno UA, Adie AP, Ibok UJ (2012). Comparative study of levels of trace Metals in moss species in some cities of the Niger Delta Region of Nigeria. International Journal of Applied Science and Technology 2(3):127-135.

Elibol B, Ezor T, Kar R, Yavali C, Colak E (2011). Antifungal and antibacterial effects of some acrocarpic mosses. African Journal of Biotechnology 10(6):986-989.

Fatoba PO, Odu EA (1999). The distribution of some tropical mosses in relation to nutrient status of substrates. Nigerian Journal of Pure and Applied Science 14:861-868.

Femi-Adepoju AG, Adepoju AO, Ogunkunle ATJ (2014). Assessment of fungicidal and fungistatic properties of some Nigerian moss plants. Global Journal of Biology, Agriculture and Health Sciences 3(1):126131.

Iihan S, Savaroglu F, Colak F, Filik-Iseen C, Erderngil FZ (2006). Antimicrobial activities of Palustriella commutate (Hedw.) Ochyra extracts (Bryophyta). Turkish Journal of Biology 30:149-152.

Ite AE, Udousoro II, Ibok UJ (2014). Distribution of some atmospheric heavy metals in lichen and moss samples collected from Eket and Ibeno Local Government Areas of Akwa Ibom State, Nigeria. American Journal of Environmental Protection 2(1):22-31.

Kathiresan P, Karar P, Siva H (2011). Comparative micromorphological and phytochemical studies on the roots of three Viburnum (Caprifoliaceae) species. Turkish Journal of Botany 35:663-670.

Kumar SS, Bassi G, Talwani S (1988). SOCGI plant chromosome number reports VI. Journal of Cytology and Genetics 23:38-52.

Makinde AM, Odu EA (1993). Re-colonization of fire disturbed soil in 
derived Savanna. Nigerian Journal of Botany 6:217-221.

McAdam SV (1982). Chromosome evolution in the Brachytheciaceae Journal of Bryology 12:233-258.

Muntung A (1974). Accessory chromosomes. Annual Review of Genetics 8:243-266.

Ojo OO, Ajayi AO, Anibijuwon II (2007). Antimicrobial of methanol extracts of lower plants. Journal of Zaejiang University Science B 8:189191.

Olorode O (1974). Chromosome numbers in Nigerian Compositae. Botanical Journal of the Linnaean Society 69:329-335.

Oyesiku OO (2012). A review of Nigerian bryophytes: past, present and future. African Journal of Agricultural Research 7(31):4352-4356.

Ramsay HP (1964). The chromosomes of Dawsonia. Bryologist 67:153162.

Ramsay HP (1969). Cytological studies on some mosses from the British Isles. Botanical Journal of the Linnaean Society 67:153-162.

Russell MD (2010). Antibiotic activity of extracts from some bryophytes in South Western British Columbia. Medical Studies Journal of Australia 2:9-14.
Savarogu F, Filik IC, Oztopen VP, Kabadere S, Iihan S, Uyar R (2011). Determination of antimicrobial and antiproliferative activities of the aquatic moss, Funtinalis antipyretica Hedw. Turkish Journal of Biology 35:361-369.

SaxenaK, HarinderS (2004). Uses of Bryophytes. Resonance 9(6):56-65.

Schofield WB (1985). Introduction to Bryology. The Blackburn Press. Cadwell, New Jersey, USA.

Smith AJE (1978). The moss flora of Britain and Ireland. Cambridge University Press, UK.

Snider JA (1973). Chromosome studies of some mosses of the Douglas Lake region III. Michigan Botany 12:107-117.

Tedela PO, Adebiyi AO, Aremu A (2014). In vitro antibacterial activity of two mosses: Calymperes erosum C. Mull and Bryum coronatum Schwaegr. from South-Western Nigeria. Journal of Biology and Life Science 5(2):77-84. 